\title{
Recurrent chylous effusions and venous thrombosis: Uncommon presentation of a common condition
}

\author{
Manasvini Bhatt ${ }^{1}$, Netto George ${ }^{1}$, Manish Soneja ${ }^{1, *}$, Prabhat Kumar ${ }^{1}$, Surabhi Vyas ${ }^{2}$, \\ Ashutosh Biswas ${ }^{1}$ \\ ${ }^{1}$ Department of Medicine, All India Institute of Medical Sciences, New Delhi, India; \\ ${ }^{2}$ Department of Radiodiagnosis, All India Institute of Medical Sciences, New Delhi, India.
}

\begin{abstract}
Summary Spontaneous bilateral chylothorax and chylous ascites rarely develop in conjunction with systemic venous thrombosis, and the most common cause of non-traumatic chylous effusion is a malignancy. A 23-year-old immunocompetent female presented with a fever of 5 months' duration associated with progressive shortness of breath and abdominal distension. Evaluation revealed bilateral chylothorax, chylous ascites, and multiple venous thrombosis. Anti-tubercular drugs were initiated on the basis of a lymph node biopsy and computed tomography findings, but her symptoms worsened, and she developed massive bilateral pleural effusions with type 2 respiratory failure requiring invasive mechanical ventilation. She was managed with anti-tubercular drugs, chest tube drainage, octreotide, anticoagulants, and other supportive treatments. A multipronged approach to the management of chylous effusions and addition of octreotide led to resolution of symptoms. The challenges faced in diagnosing and managing this case are discussed in this report.
\end{abstract}

Keywords: Chylothorax, chylous ascites, tuberculosis, hypercoagulability, octreotide

\section{Introduction}

Chylothorax and chylous ascites are characterized by accumulation of chyle in the pleural and peritoneal cavities produced by obstruction and disruption of the lymphatic channels. Chylothorax and chylous ascites are commonly caused by direct injury to the thoracic duct after surgery or by infiltration of the lymphatic system secondary to a malignant disease $(1,2)$. The reported incidence of the combined occurrence of chylothorax and chylous ascites has varied from $9 \%$ to $55 \%$ in cases of chylous effusions $(3,4)$. Tuberculosis (TB) and its association with a hypercoagulable state are seldom recognized as causes of chylothorax and chylous ascites. Presented here is the case of a young immunocompetent female with recurrent bilateral chylothorax and chylous ascites and venous thrombosis in major central veins of

Released online in J-STAGE as advance publication August 28, 2018.

*Address correspondence to:

Dr. Manish Soneja, Department of Medicine, All India Institute of Medical Sciences, New Delhi 110029, India.

E-mail:manishsoneja@gmail.com the body. These conditions were all attributed to TB, a common infection. The challenges faced in diagnosing and managing this case are discussed in this report.

\section{Case Report}

A 23-year-old female with no prior comorbidities presented with a low-grade fever (documented 100$101^{0} \mathrm{~F}$ ) of 5 months' duration associated with loss of appetite and weight loss. She also complained of progressive shortness of breath and abdominal distension for 3 months prior. An examination at a local hospital revealed bilateral chylothorax and chylous ascites. The woman was admitted there for the same complaints twice. There, she underwent multiple pleurocenteses, followed by bilateral intercostal tube insertion. A cervical lymph node was biopsied, revealing multiple welldefined caseating granulomas with necrosis. Contrastenhanced computed tomography (CECT) of the chest and abdomen suggested a tubercular etiology, and category 1 anti-tubercular therapy was initiated. The woman was then discharged. Ten days later, her symptoms worsened, so she was referred to this Hospital. An interview indicated that she had a fever for 5 months with weight 
loss, shortness of breath and abdominal distension for 3 months that worsened over the previous 10 days, and altered sensorium for 5 days. There was no history of coughing with expectoration, hemoptysis, or abnormal bowel movements.

Table 1. Routine hematological and biochemical profile

\begin{tabular}{lcc}
\hline Items & At admission & At discharge \\
\hline Hb $(\mathrm{gm} / \mathrm{dL})$ & 10.9 & 9.6 \\
Platelet count $\left(/ \mathrm{mm}^{3}\right)$ & $591 \times 10^{6}$ & $587 \times 10^{6}$ \\
TLC $\left(/ \mathrm{mm}^{3}\right)$ & 10,300 & 10,000 \\
Urea $(\mathrm{mg} / \mathrm{dL})$ & 16 & 19 \\
Creatinine $(\mathrm{mg} / \mathrm{dL})$ & 0.2 & 0.4 \\
Na $(\mathrm{mEq} / \mathrm{L})$ & 139 & 136 \\
K $(\mathrm{mEq} / \mathrm{L})$ & 4.4 & 4.3 \\
Total bilirubin $(\mathrm{mg} / \mathrm{dL})$ & 1.0 & 1.1 \\
Total protein $(\mathrm{g} / \mathrm{dL})$ & 5.7 & 6.2 \\
Albumin $(\mathrm{g} / \mathrm{dL})$ & 2.5 & 2.8 \\
Globulin $(\mathrm{g} / \mathrm{dL})$ & 3.2 & 3.4 \\
AST $(\mathrm{IU} / \mathrm{L})$ & 38 & 20 \\
ALT $(\mathrm{IU} / \mathrm{L})$ & 18 & 8 \\
ALP $(\mathrm{IU} / \mathrm{L})$ & 245 & 204 \\
ESR (mm/hr) & 52 & 34 \\
\hline
\end{tabular}

ALP, alkaline phosphatase; ALT, alanine aminotransferase; AST, aspartate aminotransferase; ESR, erythrocyte sedimentation rate; $\mathrm{Hb}$, hemoglobin; TLC, total leucocyte count.

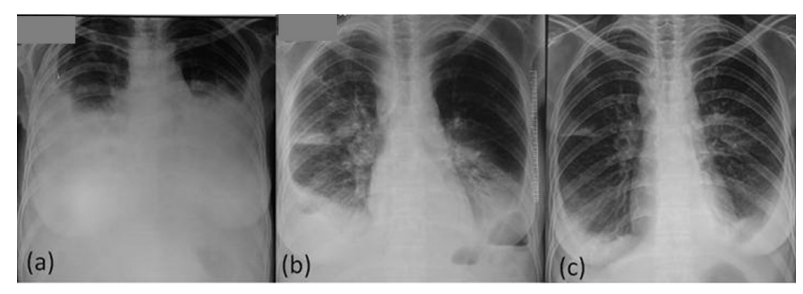

Figure 1. Serial chest radiographs. (A) At admission showing bilateral partially opaque hemithoraces and patchy opacities in bilateral lung fields; (B) Showing a reduction in bilateral lung field opacities with fluid along the bilateral costophrenic angles and right horizontal fissure; (C) Follow-up radiograph showing minimal residual opacities in the bilateral lower lung fields and costophrenic angles.
On examination, she was drowsy (10 on the Glasgow Coma Scale) and had stable vital signs. Pallor was present. A respiratory examination revealed a dull percussion note and absence of breath sounds in the bilateral inframammary, infra axillary, and infra scapular regions. There was no shifting dullness or organomegaly on abdominal examination. The rest of the general and systemic examination was normal.

A routine hematological and biochemical profile revealed normocytic normochromic anaemia, an elevated erythrocyte sedimentation rate (ESR), and reversal of the albumin:globulin ratio (Table 1). A chest radiograph revealed bilateral massive pleural effusions (Figure 1 $A$ and 1B). An intercostal tube was placed on the right side, and it drained chylous fluid with an uninterrupted flow. As her condition progressively worsened, another intercostal tube was placed on the left side. She required intubation on day 2 of hospitalization due to worsening type 2 respiratory failure. A chest radiograph was repeated to rule out consolidation, and a twodimensional (2D)-echocardiography was performed to rule out massive pulmonary embolism as a cause of the sudden worsening of the patient's condition that required mechanical ventilation. Chylous fluid was exudative in nature and predominantly consisted of lymphocytes. Triglycerides were elevated, and results of a microbiological (Gram staining, acid-fast bacilli staining/ GeneXpert/fungal staining) and a cytological evaluation were negative (Table 2). There was no evidence of ascites in an ultrasound of the abdomen. CECT of the chest and abdomen suggested bilateral pleural effusion with enlarged mediastinal and retroperitoneal lymph nodes and ileo-cecal thickening (Figure 2). CECT also revealed partial thrombosis of the right internal jugular vein, left brachiocephalic vein, and right iliac vein. Coagulation and autoimmune profiles were normal (Table 3 ). Since the patient was on mechanical ventilation, invasive sampling of the mediastinal lymph nodes was

Table 2. Analysis of pleural fluid at different time points

\begin{tabular}{lcc}
\hline Items & Sample 1 & Sample 2 (on octreotide ) \\
\hline TLC $\left(/ \mathrm{mm}^{3}\right)$ & 350 & 160 \\
DLC & L60P40 & L80P20 \\
VLDL $(\mathrm{mg} / \mathrm{dL})$ & 30 & 21 \\
Triglycerides $(\mathrm{mg} / \mathrm{dL})$ & 733 & 154 \\
Cholesterol $(\mathrm{mg} / \mathrm{dL})$ & 63 & 179 \\
LDL/HDL & 2.7 & 4.1 \\
LDL $(\mathrm{mg} / \mathrm{dL})$ & 24 & 127 \\
Amylase $(\mathrm{IU} / \mathrm{L})$ & 1.5 & 1.6 \\
Albumin $(\mathrm{g} / \mathrm{dL})$ & 1.2 & 1.8 \\
Glucose $(\mathrm{mg} / \mathrm{dL})$ & 55 & 60 \\
Protein $(\mathrm{g} / \mathrm{dL})$ & 2.3 & 2.9 \\
Gram staining/bacterial culture & No organism/Negative \\
KOH staining/fungal culture & No organism/Negative & No organism/Negative \\
AFB staining & Negative & No organism/Negative \\
GeneXpert & Negative & Negative \\
Malignant cytology & Negative & Negative \\
\end{tabular}

AFB, acid-fast bacilli; DLC, differential leucocyte count; L, lymphocytes; LDL, low-density lipoprotein; P, polymorphs; TLC, total leucocyte count; VLDL, very-low-density lipoprotein. 


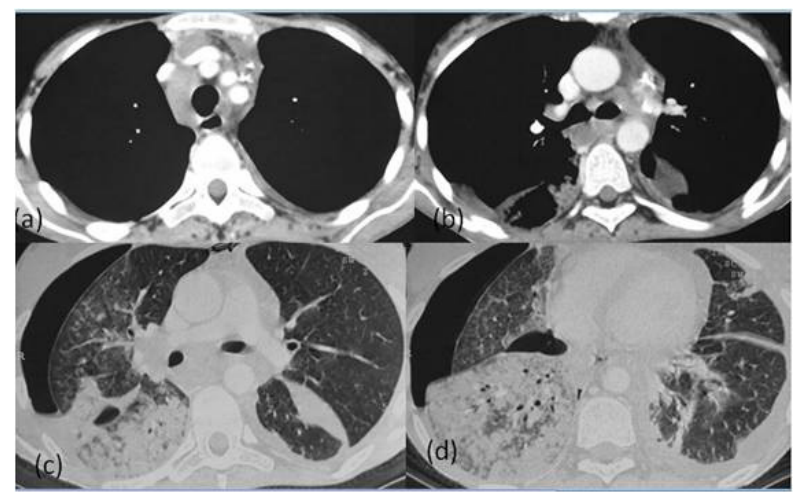

Figure 2. Axial CT images. Mediastinal windows (A, B) showing enlarged right paratracheal and aorto-pulmonary window lymph nodes with minimal bilateral pleural effusion. Lung window images $(\mathbf{C}, \mathbf{D})$ showing right-sided pneumothorax with areas of consolidation in the right lower lobe. There are centrilobular nodules with a tree-in-bud appearance in the right upper lobe.

Table 3. Results of a work-up for coagulation abnormalities

\begin{tabular}{lc}
\hline Parameter & Value \\
\hline Prothrombin time (sec) & 14.5 \\
INR & 1.5 \\
aPTT (sec) & 34 \\
Anti-cardiolipin antibody & Negative \\
Lupus anticoagulant & Negative \\
\hline
\end{tabular}

aPTT, activated partial thromboplastin time; INR, international normalized ratio.

not attempted, and anti-tubercular therapy was continued. To rule out an underlying malignancy, 18F-fluoro-deoxyglucose positron emission tomography with computed tomography (18F-FDG-PET/CT) was performed. PET/ $\mathrm{CT}$ revealed an increased FDG uptake in bilateral pleural (mediastinal and retroperitoneal) lymph nodes. A CTguided biopsy of the retroperitoneal lymph nodes was negative according to GeneXpert, and histopathology indicated predominant necrosis and few epitheloid cells. Lymphangiography was performed starting at the retroperitoneal lymph nodes and lymphoscintigraphy was performed to identify the source of lymphatic leakage, but those examinations revealed no leakage. In light of the patient's symptomatic recurrent chylous effusions, she was started on intravenous octreotide $100 \mathrm{mcg}$ three times a day. This was gradually reduced as the drain output decreased. The patient was gradually weaned off of the ventilator, and the intercostal tubes were removed. She was started on a medium-chain triglyceride-rich diet. Anticoagulation with heparin was started and coincided with warfarin as the patient's coagulation profile was monitored. The patient was hemodynamically stable, so she was discharged. Her condition was satisfactory at a follow-up 4 months after discharge (Figure 1C).

\section{Discussion}

The current patient presented with recurrent bilateral chylothorax and chylous ascites due to $\mathrm{TB}$, that also caused a systemic hypercoagulable state. Chylothorax (chylous effusion) is defined as accumulation of chyle in the pleural space due to disruption or obstruction of the thoracic duct (4). The hallmark of chylous effusion is the presence of chylomicrons in the fluid. Objective diagnostic criteria include a pleural fluid triglyceride level $>110 \mathrm{mg} / \mathrm{dL}$ and a ratio of pleural fluid to serum triglyceride level of $>1.0$. Both criteria were fulfilled in the current case.

The most common cause of non-traumatic chylous effusion is a malignancy, such as lymphoma or metastatic carcinoma $(5,6)$. Other causes of non-traumatic chylous effusion include idiopathic chylous effusion, a congenital anomaly, protein-losing enteropathy, and TB $(6,7)$. The current patient denied any history of trauma, CT scans of the chest and abdomen revealed no evidence of a malignancy, and repeated cytologic examinations of pleural effusion revealed no malignant cells. A CT scan showed multiple necrotic lymph nodes in the abdominal cavity and centrilobular nodules with a treein-bud appearance in the right lung suggestive of TB, but a fluid examination was negative for acid-fast bacilli, negative according to GeneXpert, and fluid contained low levels of adenosine deaminase. Acid-fast staining and GeneXpert testing of pleural fluid have low levels of sensitivity, and their role in examining chylous fluid has seldom been reported ( 8 ).

A possible mechanism for tuberculous lymphadenitis causing chylothorax is presumably enlarged mediastinal and hilar lymph nodes obstructing lymph flow, thereby facilitating lymphovenous communication between the thoracic duct and the azygos and intercostal veins by placing pressure on the thoracic duct and cisterna chyli (9-11). Methods used to find the site of a chylous leak like lymphangiography are not viable due to difficulty in visualizing the entire length of the thoracic duct because of the poor mix of oily contrast medium and chyle. A detailed examination of the lymphatic system was accomplished via lymphoscintigraphy, which has a sensitivity of $88 \%$ and a specificity of $100 \%$ $(12,13)$. However, it lacks sufficient spatial resolution to outline morphologic details. In the current case, lymphoscintigraphy was performed after the patient was stabilized, and it revealed no leaks. Non-contrast magnetic resonance lymphangiography allows detailed visualization of the entire thoracic duct, but its role has only been described in a few case series.

TB is associated with a systemic pro-coagulant state, as indicated by enhanced coagulation activation (increased plasma levels of thrombin-antithrombin complexes, D-dimer, and fibrinogen) along with impaired anticoagulant mechanisms like low plasma levels of antithrombin (14). Disease results in the production of pro-inflammatory cytokines that make the vascular endothelium more thrombogenic, in turn increasing the synthesis of coagulation proteins by the 
liver. TB can cause thrombosis by various mechanisms such as local invasion, venous compression, or by producing a transitory hypercoagulable state (15-17). Although TB is widely prevalent in India, data on its association with a hypercoagulable state are limited. Whether anticoagulation is needed and its duration have yet to be standardized, so carefully conducted clinical trials need to be conducted to answer these questions. Moreover, oral anticoagulation must be carefully monitored because of hepatic enzyme induction by antiTB drugs (10-12).

Treatment of chylothorax requires a multipronged approach. Conservative treatment involves supplementation of medium-chain triglycerides, total parenteral nutrition, and thoracentesis. Octreotide has been successfully used to treat chylothorax even though its dose and duration have not been clearly specified. In retrospective studies of the effectiveness of octreotide in treating chylothorax, the consensus view was that conservative treatment with octreotide should be instituted for 1 week before considering surgery $(18,19)$. In the current case, treatment with octreotide and low-molecular-weight heparin was used and chylous drainage gradually subsided. The patient was weaned off of ventilatory support and chest drains were removed. Further imaging suggested resolution of the effusion, so she was discharged. The patient's condition was satisfactory at follow up after 4 months.

In conclusion, chylous effusions with venous thrombosis can be caused by TB, although traumatic injury and malignancy are more common etiologies. Treatment of chylous effusions requires a multipronged approach, and octreotide is a useful treatment for those effusions.

\section{References}

1. Cárdenas A, Chopra S. Chylous ascites. Am J Gastroenterol. 2002; 97:1896-1900.

2. Aalami OO, Allen DB, Organ CH Jr. Chylous ascites: A collective review. Surgery. 2000; 128:761-778.

3. Romero S, Martín C, Hernandez L, Verdu J, Trigo C, Perez-Mateo M, Alemany L. Chylothorax in cirrhosis of the liver: Analysis of its frequency and clinical characteristics. Chest. 1998; 114:154-159.

4. Maldonado F, Hawkins FJ, Daniels CE, Doerr CH, Decker PA, Ryu JH. Pleural fluid characteristics of chylothorax. Mayo Clin Proc. 2009; 84:129-133.

5. Expert Panel on Vascular Imaging and Interventional Radiology: Majdalany BS, Murrey DA Jr, Kapoor BS, Cain TR, Ganguli S, Kent MS, Maldonado F, McBride JJ, Minocha J, Reis SP, Lorenz JM, Kalva SP. ACR
Appropriateness Criteria ${ }^{\circledR}$ Chylothorax Treatment Planning. J Am Coll Radiol. 2017; 14:S118-S126.

6. Hillerdal G. Chylothorax and pseudochylothorax. Eur Respir J. 1997; 10:1157-1162.

7. McGrath EE, Blades Z, Anderson PB. Chylothorax: Aetiology, diagnosis and therapeutic options. Respir Med. 2010; 104:1-8.

8. Sharma SK, Kohli M, Chaubey J, Yadav RN, Sharma R, Singh BK, Sreenivas V, Sharma A, Bhatia R, Jain D, Seenu V, Dhar A, Soneja M. Evaluation of Xpert MTB/ RIF assay performance in diagnosing extrapulmonary tuberculosis among adults in a tertiary care centre in India. Eur Respir J. 2014; 44:1090-1093.

9. El Hammoumi MM, Drissi G, Achir A, Benchekroun A, Kabiri EH, Benosman A. Spontaneous bilateral chylothorax revealing a mediastinal and abdominal lymph node tuberculosis. Rev Pneumol Clin. 2014; 70:173-176. ( in French)

10. Kutlu O, Demirbas S, Sakin A. Chylothorax due to tuberculosis lymphadenitis. North Clin Istanb. 2017; 3:225-228.

11. Rajagopala S, Kancherla R, Ramanathan RP, Tuberculosis-associated chylothorax: Case report and systematic review of the literature. Respiration. 2018; 95:260-268.

12. Kant S, Verma SK, Anand SC, Prasad R, Verma RK. Development of bilateral chylothorax in a younger female secondary to tuberculosis. Lung India. 2011; 28:56-59.

13. Weiss M, Schwarz F, Wallmichrath J, Baumeister R, Frick A, Bartenstein P, Rominger A. Chylothorax and chylous ascites. Clinical utility of planar scintigraphy and tomographic imaging with SPECT/CT. Nuklearmedizin. 2015; 54:231-240.

14. Notohamiprodjo M, Weiss M, Baumeister RG, Sommer WH, Helck A, Crispin A, Reiser MF, Herrmann KA. MR lymphangiography at 3.0 T: Correlation with lymphoscintigraphy. Radiology. 2012; 264:78-87.

15. Kager LM, Blok DC, Lede IO, et al. Pulmonary tuberculosis induces a systemic hypercoagulable state. J Infect. 2015; 70:324-334.

16. Goncalves IM , Alves DC, Carvalho A, do Ceu Brito M, Calvario F, Duarte R. Tuberculosis and venous thromboembolism: A case series. Cases J. 2009; 2:9333.

17. Gupta A, Mrigpuri P, Faye A, Bandyopadhyay D, Singla R . Pulmonary tuberculosis - An emerging risk factor for venous thromboembolism: A case series and review of literature. Lung India. 2017; 34:65-69.

18. Sharkey AJ, Rao JN. The successful use of octreotide in the treatment of traumatic chylothorax. Tex Heart Inst J. 2012; 39:428-430.

19. Ismail NA, Gordon J, Dunning J. The use of octreotide in the treatment of chylothorax following cardiothoracic surgery. Interact Cardiovasc Thorac Surg. 2015; 20:848854.

(Received July 14, 2018; Revised August 17, 2018; Accepted August 19, 2018) 